\title{
ENCLOSURE THEOREMS FOR EIGENVALUES OF ELLIPTIC OPERATORS
}

\author{
C. A. SWANSON ${ }^{1}$
}

1. Introduction. Eigenvalue problems will be considered for linear, elliptic, self-adjoint partial differential operators on $n$-dimensional Euclidean space $E^{n}$. A typical point in $E^{n}$ will be denoted by $x=\left(x^{1}, x^{2}, \cdots, x^{n}\right)$, and the Euclidean norm of $x$ by $|x|$. Partial differentiation with respect to $x^{i}$ will be denoted by $D_{i}, i=1,2, \cdots, n$. Elliptic operators $L$ defined by

$$
L u=\frac{1}{c}\left\{-\sum_{i, j=1}^{n} D_{i}\left(a_{i j} D_{j} u\right)+b u\right\}, \quad a_{i j}=a_{j i}
$$

are to be considered when the coefficients $a_{i j}, b$, and $c$ are continuous real-valued functions with $b \geqq 0, c>0$ in $E^{n}$. The ellipticity of $L$ implies that the symmetric matrix $\left(a_{i j}\right)$ is everywhere positive definite. A "solution" $u$ of $L u=0$ is supposed to be of class $C^{1}$ and all derivatives involved in (1.1) are supposed to exist, be continuous, and satisfy $L u=0$ at every point.

The eigenvalue problem for $L$ on $E^{n}$ will be called the basic problem. The only assumption to be made is that there exists at least one eigenvalue $\lambda$ for this problem whose associated eigenfunctions are " $L$-strongly asymptotic to zero" as $x \rightarrow \infty$ (definition in $\$ 2$ ). Our purpose is to obtain variational formulae for the eigenvalues and eigenfunctions of $L$ when $E^{n}$ is perturbed to an $n$-disk of large radius $a$, and the null boundary condition is adjoined on the bounding $(n-1)$ sphere. If the eigenspace of $\lambda$ is $m$-dimensional, our first theorem shows in particular that at least $m$ eigenvalues of the perturbed problem converge to $\lambda$ as $a \rightarrow \infty$. Our other results are refinements of this which lead to asymptotic estimates for eigenfunctions. The method of estimation used here is due to H. F. Bohnenblust.

The problem at hand of estimating eigenvalues and eigenfunctions for large domains has its physical origin in certain models of enclosed quantum mechanical systems, considered by a number of authors including de Groot and ten Seldam [2], [12], Dingle [3], Hull and Julius [6], Sommerfeld and Hartman [7]. In the case that the Schrödinger operator (a special case of (1.1)) is separable, the problem reduces to a domain-perturbation problem for a singular second-

Received by the editors January 26, 1965.

1 This research was supported by the United States Air Force Office of Scientific Research, under grant AF-AFOSR-379-63. 
order ordinary differential operator, for which various results have been obtained [8], [9], [10].

2. Basic and perturbed problems. Let $R_{a}$ denote the $n$-disk $\{x:|x|<a, a>0\}$, and let $B=B_{a}$ denote the bounding $(n-1)$-sphere. Let $\mathfrak{S}, \mathfrak{S}_{a}$ be the Hilbert spaces which are the Lebesgue spaces with respective inner products defined by

$$
\langle u, v\rangle=\int_{E^{n}} u(x) \bar{v}(x) c(x) d x, \quad\langle u, v\rangle_{a}=\int_{R_{a}} u(x) \bar{v}(x) c(x) d x .
$$

The Hilbert norms will be denoted as usual by $\|u\|,\|u\|_{a}$.

The basic eigenvalue problem for $L$ is

$$
L u=\lambda u, \quad u \in \mathfrak{S},
$$

where any eigenfunction is a solution of the differential equation in the sense described in $\$ 1$.

The perturbed domain $\mathfrak{D}_{a}$ is defined as the set of all complex-valued functions $v$ which satisfy the following conditions:

(i) $v$ is continuous on $\bar{R}_{a}$;

(ii) $v$ has uniformly continuous first partial derivatives in $R_{a}$;

(iii) all derivatives of $v$ involved in $L v$ exist and are continuous in $R_{a}$;

(iv) $v$ vanishes on $B_{a}$.

The perturbed eigenvalue problem under consideration is

$$
L v=\mu v, \quad v \in \mathfrak{D}_{a} .
$$

It is known [1], [5] on account of the ellipticity of $L$ on $\bar{R}_{a}$ that there exists a denumerable set of eigenvalues $\left\{\mu_{j}\right\}\left(0<\mu_{1} \leqq \mu_{2} \leqq \cdots\right)$ and a corresponding complete orthonormal sequence of eigenfunctions $v_{j}$. Green's function $K_{a}(x, y)$ is constructed in the usual way as the sum of a fundamental solution of $L v=0$ and the solution of a suitable Dirichlet problem. If $K_{a}$ is the linear integral operator whose kernel is Green's function, the eigenfunctions $v_{j}$ satisfy the integral equation

$$
v_{j}=\mu_{j} K_{a} v_{j}, \quad j=1,2, \cdots,
$$

and any basic eigenfunction $u$ satisfies $L K_{a} u=u$ in $R_{a}$ [1].

A parametrix is a nondecreasing continuous function $\phi$ in $0<a<\infty$ such that $\lim \phi(a)=\infty(a \rightarrow \infty)$. An L-indicator is a solution $G$ of the differential equation $L G=0$ in $E^{n}$ which is uniformly asymptotic to some parametrix $\phi$ as $x \rightarrow \infty$, i.e. $G(x) \sim \phi(|x|)$ uniformly in $E^{n}$. A function $u$ is said to be L-strongly asymptotic to zero if there exists an $L$-indicator such that 


$$
\lim _{|x| \rightarrow \infty} u(x)\|G\|_{|x|}\left(G(x)\|u\|_{|x|}\right)^{-1}=0
$$

uniformly in $E^{n}$.

It is not true in general that the eigenvalues $\mu=\mu(a)$ of $(2.2)$ tend to limits as $a \rightarrow \infty$, even when the spectrum of the basic problem is entirely discrete. Easy counterexamples are provided in the case $n=1$ when the singularity at $\infty$ is of the limit circle type in Weyl's classification [9]. Here we shall prove the convergence of the eigenvalues $\mu(a)$ to basic eigenvalues under the assumption that all basic eigenfunctions are $L$-strongly asymptotic to zero.

As an example of (1.1), consider the Schrödinger operator $L=$ $-\Delta+b$, where $b(x)=|x|^{2}+o(1)$ as $|x| \rightarrow \infty$. Since $b(x) \rightarrow \infty$, the whole basic spectrum is discrete $[13$, p. 150]. A parametrix is $\phi(a)$ $=a^{-n / 2} \exp \left(a^{2} / 2\right), a>\sqrt{ } n / 2$, and every basic eigenfunction satisfies (2.4).

3. Asymptotic estimates for eigenvalues. The eigenspace associated with a basic eigenvalue $\lambda$ will be denoted by $\mathfrak{A}_{\lambda}$. The following notations will be used

$$
\begin{aligned}
\psi_{a}[u] & =2\left(\max _{B}|u|\right)\|G\|_{a}\left(\phi(a)\|u\|_{a}\right)^{-1} \quad(u \neq 0) \\
\psi_{a} & =\sup _{u \in \mathfrak{T}_{\lambda}} \psi_{a}[u] ; \quad \rho_{a}=2 \lambda \psi_{a} /\left(1-2 \psi_{a}\right) .
\end{aligned}
$$

Since every $u \in \mathfrak{A}_{\lambda}$ has the form $u=\sum_{i=1}^{m} \alpha_{i} u_{i}$ in terms of an orthonormal basis $\left\{u_{i}\right\}$, it is easy to verify that

$$
\psi_{a} \leqq 2 m \max _{1 \leqq i \leqq m} \psi_{a}\left[u_{i}\right]
$$

It follows from (2.4) that $\psi_{a}=o(1)$ and $\rho_{a}=o(1)$ as $a \rightarrow \infty$.

THEOREM 1. If $\lambda$ is a basic eigenvalue possessing $m$ orthonormal eigenfunctions which are L-strongly asymptotic to zero, there exists a positive number $a_{0}$ such that at least $m$ perturbed eigenvalues $\mu_{i}(a)$ of $(2.2)$ are enclosed in the interval $\left[\lambda, \lambda+\rho_{a}\right]$ whenever $a \geqq a_{0}$.

Proof. It follows from the maximum principle for elliptic differential equations $\left[1\right.$, p. 326] that $\lambda>0$. Let $\alpha=1 / \lambda$. For every $u \in \mathfrak{A}_{\lambda}$, the function $f=K_{a} u-\alpha u$ is the solution of the Dirichlet problem $L f=0$ in $R_{a}, f=-\alpha u$ on $B_{a}$. Define

$$
g(x)=G(x) / \phi(a) ; \quad F(x)=2\left(\max _{B}|f|\right) g(x)-f(x) .
$$


Since $G(x) \sim \phi(|x|)$ as $x \rightarrow \infty$, there exists a positive number $a_{0}$ such that $g(x) \geqq \frac{1}{2}$ on $B_{a}$ and $2 \psi_{a}<1$ whenever $a \geqq a_{0}$. Hence $F(x) \geqq 0$ on $B_{a}$, and it follows from the maximum principle that $F(x) \geqq 0$ throughout $R_{a}$, or

$$
f(x) \leqq 2\left(\max _{B}|f|\right) g(x), \quad x \in R_{a}
$$

Similarly

$$
f(x) \geqq-2\left(\max _{B}|f|\right) g(x), \quad x \in R_{a},
$$

and consequently

$$
\|f\|_{a} \leqq 2 \alpha\left(\max _{B}|u|\right)\|G\|_{a} / \phi(a)
$$

or by $(3.1)$,

$$
\left\|K_{a} u-\alpha u\right\|_{a} \leqq 2 \alpha \psi_{a}\|u\|_{a} .
$$

Let $P(\epsilon)$ be the projection operator from $\mathfrak{S}_{a}$ onto the subspace $\mathfrak{F}_{a \epsilon}$ spanned by all eigenfunctions of $K_{a}$ whose corresponding eigenvalues $\beta_{i}$ lie in the interval $|\beta-\alpha|<\epsilon$. The following inequality is valid for arbitrary $u \in \mathfrak{A}_{\lambda}$

$$
\|u-P(\epsilon) u\|_{a} \leqq \epsilon^{-1}\left\|K_{a} u-\alpha u\right\|_{a} .
$$

The proof given in [11] for self-adjoint transformations extends without change to $K_{a}$. Then (3.3) yields the inequality

$$
\|u-P(\epsilon) u\|_{a} \leqq 2 \alpha \psi_{a} \epsilon^{-1}\|u\|_{a},
$$

which implies that at least $m$ eigenvalues $\beta_{i}$ of $K_{a}$ are included in the interval $\left|\beta_{i}-\alpha\right| \leqq 2 \alpha \psi_{a}, i=1,2, \cdots\left[11\right.$, p. 35]. Since $\alpha, \beta_{i}$ are reciprocals of $\lambda, \mu_{i}$ respectively, at least $m$ eigenvalues $\mu_{i}$ of the perturbed problem (2.2) satisfy $\left|\mu_{i}-\lambda\right| \leqq 2 \mu_{i} \psi_{a}$. Since $\mu_{i} \geqq \lambda$ is a general consequence of the minimax principle for eigenvalues [1], $\lambda \leqq \mu_{i} \leqq \lambda$ $+2 \mu_{i} \psi_{a}$, or $\lambda \leqq \mu_{i} \leqq \lambda /\left(1-2 \psi_{a}\right)=\lambda+\rho_{a}$, where $\rho_{a}$ is defined by (3.1). This completes the proof of Theorem 1 .

THEOREM 2. Let $\lambda$ be a basic eigenvalue of multiplicity $m$ whose eigenfunctions are all L-strongly asymptotic to zero. If there exists a basic eigenvalue exceeding $\lambda$, then there is a positive number $a_{1}$ such that exactly $m$ perturbed eigenvalues $\mu_{i}$ are enclosed in the interval $\left[\lambda, \lambda+\rho_{a}\right]$ whenever $a \geqq a_{1}$. 
Proor. Suppose first that $\lambda$ is the smallest basic eigenvalue. Let $\lambda^{\prime}$ be the smallest eigenvalue exceeding $\lambda$. Since $\psi_{a}=o(1)$ as $a \rightarrow \infty$, there is a number $a_{1} \geqq a_{0}$ such that $\psi_{a}<\left(\lambda^{\prime}-\lambda\right) / 2 \lambda^{\prime}$ whenever $a \geqq a_{1}$, which implies $\lambda+\rho_{a}<\lambda^{\prime}$. Then theorem 1 shows that at least $m$ eigenvalues $\mu_{i}$ are included in the subinterval $\left[\lambda, \lambda+\rho_{a}\right]$ of $\left[\lambda, \lambda^{\prime}\right]$. Since $\mu_{i} \geqq \lambda_{i}$ for each $i$ is a general consequence of the minimax property of eigenvalues, at most $m$ eigenvalues $\mu_{i}$ lie in this subinterval, and hence exactly $m$. If $\lambda=\lambda^{i}$ is the $i$ th distinct basic eigenvalue, $\lambda^{1}$ $<\lambda^{2}<\cdots$, an easy induction proof establishes the same result.

4. Uniform estimates for eigenfunctions. Let $p=p(n)$ be a positive number satisfying $p(2)=0, p(3)=0$, and $0<n-2 p<4$. Because the fundamental singularity of $K_{a}(x, y)$ is of order $|x-y|^{2-n}, n \geqq 3$, the function

$$
k_{a}(x)=\left(\int_{R^{a}}|x-y|^{2 p} K_{a}^{2}(x, y) c(y) d y\right)^{1 / 2}
$$

is well-defined in $R_{a}$. Our assumption for the next theorem is that

$$
\psi_{a}^{q} k_{a}(x)=o(1) \quad \text { as } \quad a \rightarrow \infty \quad(q=(n-2 p) / n)
$$

uniformly in $R_{a}$, where $\psi_{a}$ is defined by (3.1). In the case $n=1, p=0$ considered in [10, p. 310], $k_{a}(x)$ is uniformly bounded in $R_{a}$ for $a \geqq a_{0}$, and accordingly (4.1) is implied by (2.4).

Theorem 3. Corresponding to the eigenvalues $\lambda$ and $\mu_{i}$ of Theorem 2, there are orthonormal eigenfunctions $u_{i}$ associated with $\lambda$ and $v_{i}$ associated with the $\mu_{i}$ such that

$$
\begin{aligned}
& v_{i}(x)=u_{i}(x)-f_{i}(x)+O\left(\psi_{a}^{q}\right) k_{a}(x), \\
& i=1,2, \cdots, m ; \quad x \in R_{a} ; \quad a \geqq a_{1},
\end{aligned}
$$

where $f_{i}$ is the solution of the Dirichlet problem $L f_{i}=0$ in $R_{a}, f_{i}=u_{i}$ on $B_{a}$.

Proof. Select the number $\epsilon$ in (3.4) to be $\alpha-\alpha^{\prime}$, where $\alpha=1 / \lambda$, $\alpha^{\prime}=1 / \lambda^{\prime}$. With $a_{1}$ as in Theorem 2 , it follows that $2 \alpha \psi_{a}<\alpha\left(\lambda^{\prime}-\lambda\right) / \lambda^{\prime}$ $=\alpha-\alpha^{\prime}=\epsilon$ for $a \geqq a_{1}$. Then $\mathfrak{F}_{a \epsilon}$ is $m$-dimensional by Theorem 2 and $P(\epsilon) u=0$ implies $u=0$ by (3.4). Hence there exist $m$ uniquely determined linearly independent eigenfunctions $z_{i}$ corresponding to $\alpha$ which $P(\epsilon)$ maps into the orthonormal eigenfunctions $v_{i}$, and by (3.4), $\left\|z_{i}-v_{i}\right\|_{a}=O\left(\psi_{a}\right)$. Since

$$
\left|\left\langle z_{i}, z_{j}\right\rangle_{a}-\left\langle v_{i}, v_{j}\right\rangle_{a}\right| \leqq\left\|v_{i}\right\|\left\|_{a}\right\| z_{j}-v_{j}\left\|_{a}+\right\| z_{j}\|\|_{a}\left\|z_{i}-v_{i}\right\|_{a}
$$


by the Schwarz inequality, $\left\langle z_{i}, z_{j}\right\rangle_{a}=\delta_{i j}+O\left(\psi_{a}\right), i, j=1,2, \cdots, m$. Since the $z_{i}$ are linearly independent, an orthonormal sequence $\left\{u_{i}\right\}$ can be constructed by the Schmidt process as linear combinations of the $z_{i}$, and it is seen without difficulty that $\left\|u_{i}-z_{i}\right\|_{a}=O\left(\psi_{a}\right)$. Hence

$$
\left\|u_{i}-v_{i}\right\|_{a}=O\left(\psi_{a}\right), \quad i=1,2, \cdots, m .
$$

Omitting the subscripts $i$, we select a typical $u$ in the set $\left\{u_{i}\right\}$ and corresponding $v$ in the set $\left\{v_{i}\right\}$. Since $\mu-\lambda=O\left(\psi_{a}\right)$ by Theorem 2 and $\|v-u\|_{a}=O\left(\psi_{a}\right)$ by $(4.3)$, we obtain

$$
\|\mu v-\lambda u\|_{a} \leqq \mu\|v-u\|_{a}+(\mu-\lambda)\|u\|_{a}=O\left(\psi_{a}\right) .
$$

Define

$$
w_{a}(x)=\left(\int_{R_{a}}|x-y|^{-2 p}|\mu v(y)-\lambda u(y)|{ }^{2} c(y) d y\right)^{1 / 2} .
$$

Let $S(x, \delta)$ denote the $n$-disk of centre $x$ and radius $\delta$. A routine decomposition of the integral into integrals over $S(x, \delta) \cap R_{a}$ and the remainder of $R_{a}$ yields

$$
w_{a}^{2}(x) \leqq \delta^{-2 p}\|\mu v-\lambda u\|_{a}^{2}+O\left(\delta^{n-2 p}\right) .
$$

With the choice $\delta=\psi_{a}^{2 / n}$ we obtain the uniform estimate $w_{a}(x)$ $=O\left(\psi_{a}^{q}\right)$, where $0<q=(n-2 p) / n<4 / n$. In particular, $w_{a}(x)=O\left(\psi_{a}\right)$ if $n=2$ or 3 . Since

$$
v(x)-\lambda K_{a} u(x)=K_{a}(\mu v-\lambda u)(x),
$$

it follows from Schwarz's inequality that

$$
\left|v(x)-\lambda K_{a} u(x)\right| \leqq k_{a}(x) w_{a}(x)=O\left(\psi_{a}^{q}\right) k_{a}(x) .
$$

The function $g$ defined by

$$
g(x)=\lambda K_{a} u(x)-u(x)+f(x)
$$

is a solution of the Dirichlet problem $L g=0$ in $R_{a}, g=0$ on $B_{a}$, and hence $g$ is identically zero. The uniform estimate (4.2) is then a consequence of (4.4) and (4.5).

5. Asymptotic variational formulae for eigenvalues. We shall require Green's symmetric identity in the form [4]

$$
\langle L u, v\rangle_{a}-\langle u, L v\rangle_{a}=[u v]_{a}-[v u]_{a}=\{u v\}_{a},
$$

where 


$$
[u v]_{a}=\int_{B_{a}} u \sum_{k, j=1}^{n} a_{k j} n_{k} D_{j} \bar{v} d S
$$

and $\{u v\}_{a}$ is defined by (5.1). Here $\left(n_{k}\right)$ is the outward pointing unit normal to $B_{a}$.

Let $u, v$ be normalized eigenfunctions associated with $\lambda, \mu$, as described in Theorems 2 and 3. Let $f$ be the solution of the Dirichlet problem $L f=0$ in $R_{a}, f=u$ on $B_{a}$.

Since $v=0$ on $B_{a},[v u]_{a}=0$. Then application of (5.1) to the differential equations $L u=\lambda u$ and $L v=\mu v$ leads to the formula

$$
(\lambda-\mu)\langle u, v\rangle_{a}=[u v]_{a} .
$$

It is a consequence of (4.3) that

$$
\left|\langle u, v\rangle_{a}-\langle u, u\rangle_{a}\right| \leqq\|u\|_{a}\left\|_{v}-u\right\|_{a}=O\left(\psi_{a}\right) .
$$

Hence $\langle u, v\rangle_{a}=1+O\left(\psi_{a}\right)$ and (5.2) yields

$$
\lambda-\mu=[u v]_{a}\left[1+O\left(\psi_{a}\right)\right] .
$$

Application of (5.1) to the differential equations $L u=\lambda u, L v=\mu v$, and $L f=0$ leads to

$$
\begin{aligned}
& -\mu\langle f, v\rangle_{a}=[f v]_{a}=[u v]_{a} \\
& -\lambda\langle f, u\rangle_{a}=\{f u\}_{a} .
\end{aligned}
$$

Since $\mu=\lambda+O\left(\psi_{a}\right)$ by Theorem 2, it follows from (5.3) and (5.4) that

$$
\lambda-\mu=-\lambda\langle f, v\rangle_{a}\left[1+O\left(\psi_{a}\right)\right] .
$$

Finally we appeal to the uniform estimate (4.2) and to (5.5) to obtain

$$
\lambda-\mu=\left[\{f u\}_{a}-\lambda\langle f, f\rangle_{a}\right]\left[1+O\left(\psi_{a}\right)\right]+\left\langle f, k_{a}\right\rangle_{a} O\left(\psi_{a}^{q}\right) .
$$

In some cases the first term dominates the other terms, and we obtain the asymptotic form

$$
\mu(a)-\lambda \sim\{u f\}_{a} \text { as } a \rightarrow \infty .
$$

The results of Theorems 1-3 are then sharpened accordingly.

In the example considered at the end of $\$ 2$, some of the basic eigenfunctions are asymptotic to radial functions $R(|x|)$ (explicit formulae in [13]). In such cases, $f(x) \sim R(a) \phi(|x|) / \phi(a)$, and (5.6) yields the eigenvalue variation $\mu(a)-\lambda \sim \omega_{n-1} a^{n}|R(a)|^{2}$, where $\omega_{n-1}$ denotes the volume of the unit $(n-1)$-sphere. 


\section{REFERENCES}

1. R. Courant and D. Hilbert, Methods of mathematical physics, Vol. II, Interscience, New York, 1962.

2. S. R. de Groot and C. A. Seldam, On the energy levels of a model of the compressed hydrogen atom, Physica 12 (1946), 669-682.

3. R. B. Dingle, The solution of the Schrödinger equation for finite systems, with special reference to the motion of electrons in Coulomb electric fields and uniform magnetic fields, Proc. Cambridge, Philos. Soc. 49 (1953), 102-114.

4. G. F. D. Duff, Partial differential equations, Univ. Toronto Press, Toronto, 1956.

5. Lars Garrding, On the asymptotic distribution of the eigenvalues and eigenfunctions of elliptic differential operators, Math. Scand. 1 (1953), 237-255.

6. T. E. Hull and R. S. Julius, Enclosed quantum mechanical systems, Canad. J. Phys. 34 (1956), 914-919.

7. A. Sommerfeld and H. Hartman, Künstliche Grenzbedingungen in der Wellenmechanik. Der beschränkte Rotator, Ann. Phys. (5) 37 (1940), 333-343.

8. C. A. Swanson, Differential operators with perturbed domains, J. Rational Mech. Anal. 6 (1957), 823-846.

9. - Asymptotic estimates for limit circle problems, Pacific J. Math. 11 (1961), 1549-1559.

10. - Asymptotic estimates for limit point problems, Pacific J. Math. 13 (1963), 305-316.

11. - On spectral estimation, Bull. Amer. Math. Soc. 68 (1962), 33-35.

12. C. A. ten Seldam and S. R. de Groot, On the ground state of a model for compressed helium, Physica 18 (1952), 891-904.

13. E. C. Titchmarsh, Eigenfunction expansions associated with second order differential equations, Part II, Oxford Univ. Press, Oxford, 1958.

The University of British Columbia 